\title{
Zusammenfassung der empirischen Ergebnisse
}

Gesamtüberblick: $\mathrm{Zu}$ der zusammenfassenden Darstellung der unterschiedlichen Ergebnisse und zur Interpretation der Analyse zur Konzeptualisierung des Instruments zur sprachlichen Variation finden nachfolgend eine vergleichende Darstellung der Ergebnisse und eine Erörterung der inhaltlichen Erkenntnisse statt (Abschnitt 10.1). Aufgrund der zum Teil neuen methodischen Zugänge zur Untersuchung von Sprache im Mathematikunterricht bietet es sich außerdem an, die methodischen Erkenntnisse dieser Arbeit zu schildern (Abschnitt 10.2).

\subsection{Vergleichende Darstellung der Ergebnisse und inhaltliche Erkenntnisse}

Im Hinblick auf die dreiteilige Analyse dieser Arbeit ergeben sich verschiedene Ebenen der empirischen Erkenntnisgewinnung für das Instrument zur sprachlichen Variation von Textaufgaben.

Zunächst zeigen die Ergebnisse der ersten quantitativen Analyse, dass sich ein quantitativer Zugang zur Analyse von Sprache im Mathematikunterricht eignet. Durch die Quantifizierung von Textmerkmalen konnten zusätzliche Informationen über die Beziehungsstruktur von Textmerkmalen in mathematischen Textaufgaben gewonnen werden. Diese Beziehungsstrukturen wurden durch eine explorative Faktorenanalyse untersucht und die Faktoren können als Basis für Variationen von Textmerkmalen genutzt werden. Die Analyse hat damit dargelegt, dass sich systematische Muster der Verwendung von Textmerkmalen in Textaufgaben der Mathematik zeigen. Diese Musterbildung von Textmerkmalen deutet auf eine zugrunde liegende Assoziation zwischen Form und Funktion hin, die als grundlegend für die Formulierung von mathematischen Textaufgaben gedeutet werden

D. Bednorz, Sprachliche Variationen von mathematischen Textaufgaben, Bielefelder Schriften zur Didaktik der Mathematik 5, https://doi.org/10.1007/978-3-658-33003-3_10 
kann. Die Besonderheiten der Beziehungsstrukturen konnten aus diesem Grund anhand sprachlich-funktionaler Gesichtspunkte und typischer Vertextungsmuster der Exploration, Deskription und Instruktion interpretiert und bezeichnet werden. Mithin baut das Instrument zur sprachlichen Variation auf den in mathematischen Textaufgaben vorhandenen Mustern des Gebrauchs auf und können als Grundlage für zukünftige sprachliche Veränderungspraktiken genutzt werden.

Bei der zweiten quantitativen Untersuchung wurden die Faktoren des Instruments genutzt, um die Aufgabenschwierigkeiten von Testaufgaben zu schätzen und den Effekt der Faktoren auf die Aufgabenschwierigkeiten zu bestimmen.

Insgesamt zeigen die Ergebnisse der zweiten quantitativen Analyse, dass das Instrument dazu geeignet ist, die Schwierigkeit von Testaufgaben zu schätzen. Es gelingt, die Aufgabenschwierigkeit von mittelschwierigen bzw. schwierigen Aufgaben zu schätzen. Weniger genau lassen sich die Aufgabenschwierigkeiten von leichten Aufgaben schätzen. Auch der Effekt auf die Aufgabenschwierigkeit der Faktoren konnte ermittelt werden. Insgesamt drei Faktoren zeigen einen positiven Effekt auf die Aufgabenschwierigkeit, wobei der erklärende Faktor den deutlichsten positiven Einfluss hat. Moderate positive Effekt auf die Aufgabenschwierigkeit zeigen der informative und der instruktive Faktor. Geringe negative Effekte auf die Aufgabenschwierigkeit hat der beschreibende Faktor, während der komprimierende Faktor einen moderaten negativen Effekt auf die Aufgabenschwierigkeit aufweist. Die Ergebnisse können als Indiz dafür betrachtet werden, dass die Darstellungsvernetzung eine hohe Bedeutung beim Lernen von fachlichem Vokabular haben kann. Um jedoch genauere Kenntnisse darüber zu erlangen, welche Faktoren unter kontrollierten Bedingungen einen Effekt zeigen und wie sich ein strukturiertes Vorkommen der Faktoren auf die Schätzung der Aufgabenschwierigkeit auswirkt, benötigt es eine systematische Testentwicklung mithilfe des Instruments zur sprachlichen Variation von mathematischen Textaufgaben. Zunächst können die Ergebnisse aber als erster Hinweis auf den Einfluss von sprachlichen Faktoren auf die Aufgabenschwierigkeit gedeutet und dahingehend interpretiert werden, dass sich das Instrument effektiv einsetzen lässt, um die Aufgabenschwierigkeit von mathematischen Testaufgaben zu bestimmen und gegebenenfalls auch zu verändern.

Anders als bei der ersten und zweiten quantitativen Analyse ist bislang noch keine direkte Vernetzung zwischen erster quantitativer und qualitativer Untersuchung geschehen. Für die Bildung von Aufgabentypen war die Faktorenanalyse zwar bedeutsam, um charakteristische Textaufgaben auszuwählen und somit fachliche und kontextuelle Besonderheiten herauszufinden, jedoch ist ebenfalls ein theoretischer und empirischer Vergleich beider Ergebnisse möglich. Werden beide Ergebnisse auf theoretischer Basis miteinander in Beziehung gesetzt, lassen sich 
die empirischen Ergebnisse auf die theoretischen Aspekte in Abschnitt 3.3.2 und Abbildung 3.2 extrapolieren. Das Ergebnis ist in Abbildung 10.1 dargestellt. Hierin sind die empirischen Ergebnisse in dem Modell der Beziehung zwischen Sprache und Kontext nach Halliday (2007) abgetragen. Die sprachlichen Faktoren sind entlang des Pols der Sprache angeordnet. Die Aufgabentypen sind entlang des Kontextes dargestellt. Beide Ebenen stehen in Beziehung zueinander und die sprachlichen Veränderungen beziehen sich ebenfalls auf die Veränderung von (fachlichen) kontextuellen Merkmalen. Die Ergebnisse machen deutlich, dass es möglich ist, durch ein quantitatives und qualitatives Vorgehen sowohl sprachliche als auch kontextuelle Merkmale miteinander in Beziehung zu bringen und somit das Registerkonzept für kleinere Lerneinheiten - wie in diesem Fall für mathematische Textaufgaben - anzuwenden. Gleichzeitig kann das Instrument zur sprachlichen Variation genutzt werden, um neben den sprachlichen Faktoren auch inhaltliche und kontextuelle Kriterien $\mathrm{zu}$ beachten, die aus mathematikdidaktischer Perspektive und dem Blickwinkel einer mathematischen Lehrkraft bedeutsam sind.

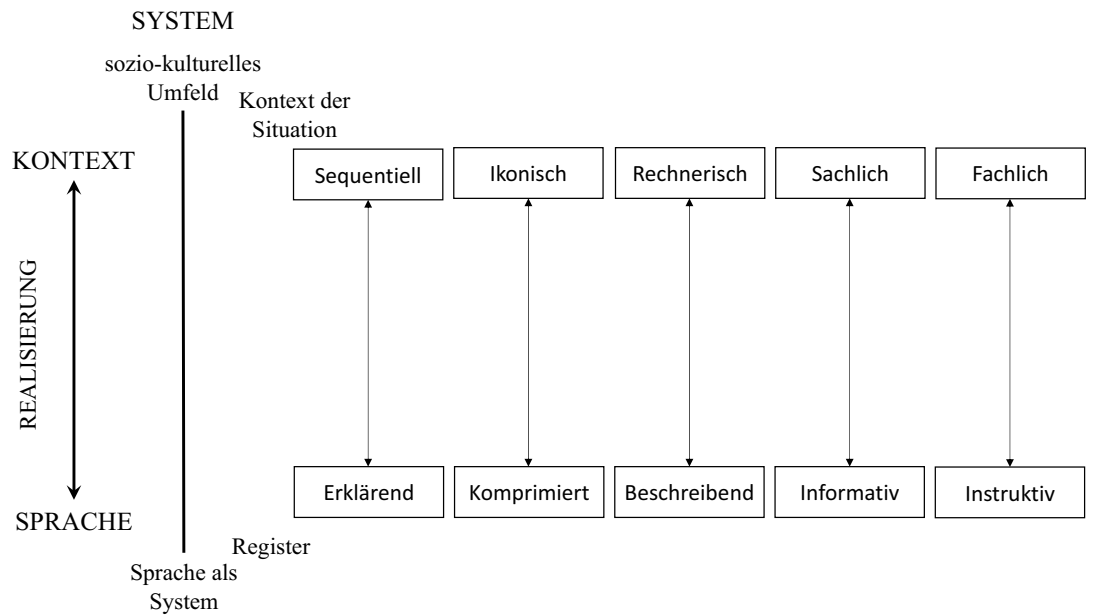

Abbildung 10.1 Theoretische Verknüpfung der ersten quantitativen und ersten qualitativen Analyse im Hinblick auf das Beziehungsmodell von Sprache und Kontext (Eigene Erstellung) 
Die zweite Möglichkeit, die Ergebnisse der beiden Analysen direkt miteinander in Beziehung zu setzen, ist ein Vergleich der Ausprägungen der durchschnittlichen Faktorenwerte jedes Aufgabentyps je Faktor. Dieser Vergleich ist in Abbildung 10.2 dargestellt. Die Abbildung zeigt, dass jeweils die Aufgabentypen einen hohen durchschnittlichen Faktorenwert auf einen Faktor zeigen, die charakteristisch für diesen Faktor sind (jeweils über eine Standardabweichung). So sind generell sequenzielle Aufgaben erklärend, sachliche Aufgaben informativ, rechnerische Aufgaben beschreibend, ikonische Aufgaben komprimierend und fachliche Aufgaben instruktiv. Darüber hinaus kann in Abbildung 10.2 gezeigt werden, dass sich Gegensatzpaare bei den Aufgabentypen bilden, die sich im durchschnittlichen Faktorenwert je Faktor deutlich unterscheiden. So zeigt sich für sequenzielle Aufgaben, dass sie nicht informativ sind. Sowohl der erklärende als auch der informative Faktor wurden in Abschnitt 7.2.5 der Explikation zugeordnet. Das bedeutet, dass die beiden möglichen Explikationsstrategien nicht gemeinsam verwendet werden, sondern entweder mehr erklärende Explikation und weniger informative Explikation genutzt wird oder umgekehrt. Dieselbe deutliche Tendenz ist für sachliche Aufgaben nicht erkennbar. Diese Aufgaben sind informativ, aber nicht instruktiv. Das gleiche Gegensatzpaar zeigt sich auch für fachliche Aufgaben. Diese sind instruktiv und nicht informativ. Der Gegensatz zwischen Explikation, also der erklärenden Formulierung, und der Instruktion, der Fokussierung auf eine Aufgabenanforderung, ist klar und zeigt sich auch noch bei sequenziellen Aufgaben deutlich.

In Anbetracht von rechnerischen Aufgaben zeigt sich, dass diese Aufgaben nicht komprimierend sind. Wie in Abschnitt 7.2.5 erörtert, lassen sich der komprimierende und der beschreibende Faktor nach der Interpretation der Deskription zuordnen. Für ikonische Aufgaben zeigt sich, dass diese komprimierend sind und nicht beschreibend. Das zeigt für die rechnerischen und ikonischen Aufgaben, dass jeweils ein Modus der Deskription zur Vermittlung der Textaufgabe verwendet wird.

Die zusammenfassende und verknüpfende Darstellung der Ergebnisse macht deutlich, welche Aspekte das Instrument zur sprachlichen Variation von mathematischen Textaufgaben umfasst, dabei werden die unterschiedliche Vertextungsmöglichkeiten in eine sinnvolle Beziehung gesetzt. Die Herstellung der Beziehung wird durch eine explorative empirische Untersuchung durchgeführt und interpretativ bezeichnet. Die Möglichkeit der sinnvollen Verknüpfung der Ergebnisse, wie in Abbildung 10.2 geschehen, kann als Indiz für die empirische Aussagekraft der Ergebnisse interpretiert werden. 


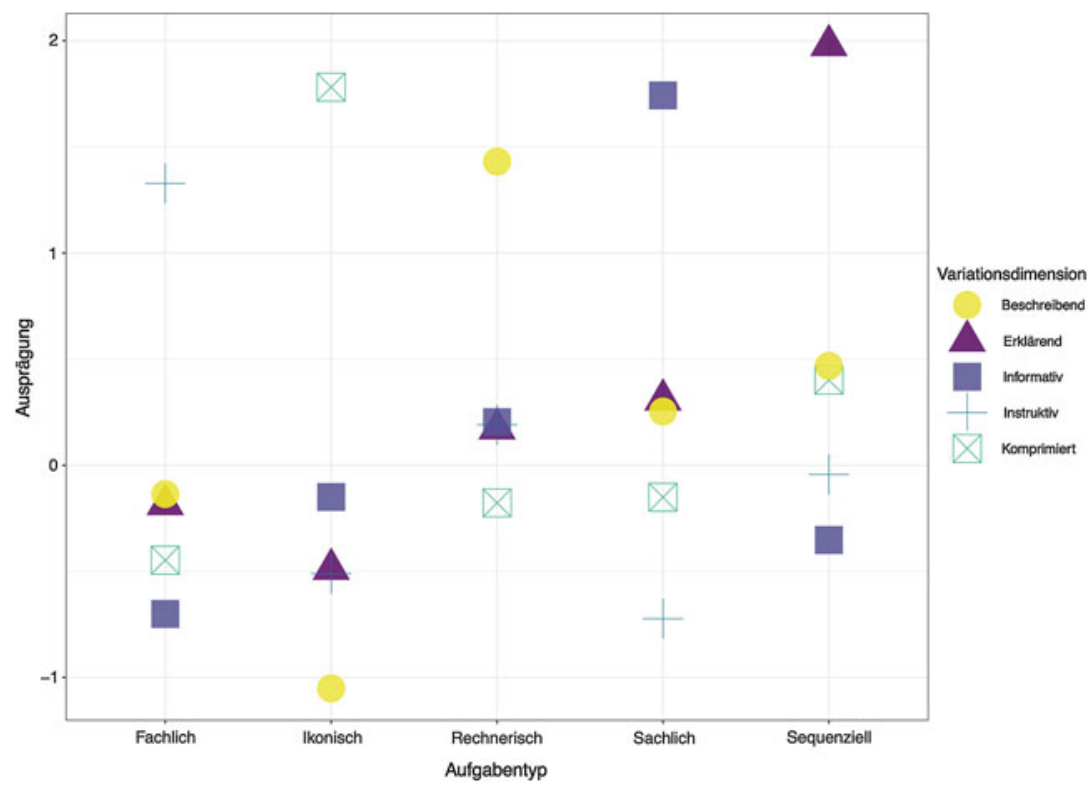

Abbildung 10.2 Ausprägungen und Vergleich der Aufgabentypen für die einzelnen Faktoren (Eigene Erstellung)

\subsection{Methodische Erkenntnisse}

Zuerst hat die Konzeptualisierung des Instruments gezeigt, dass Korpusanalysen ein erfolgreiches Verfahren darstellen, um sprachliche Merkmale zu analysieren. Das Verfahren ist besonders von Interesse, da die tatsächlich und häufig vorkommenden Textmerkmale in Textaufgaben empirisch abgebildet werden können, sodass zwischen unterschiedlichen Disziplinen, die sich im Vorkommen der Textmerkmale prinzipiell deutlich unterscheiden können, differenziert werden kann.

Des Weiteren kann die Verwendung von Korpusanalysen zur Untersuchung von produzierten Texten in Interview- oder Erhebungssituationen von Lernenden dienen. Hierdurch können Sprachstrukturen analysiert werden, die beispielsweise von bestimmten Kohorten in der Stichprobe im Vergleich zu anderen unterschieden werden. Diese Unterschiede können dazu beitragen, die heterogenen 
sprachlichen Voraussetzungen zu bestimmen und geeignete Strategien zu entwickeln, um Differenzen zu reduzieren und durch gezielte Maßnahmen Sprache aufzubauen. Auch eine Forcierung von sprachlichen Analysen, die themenspezifisch unterschieden werden, ist eine Möglichkeit, fachsprachliche Spezifika im Mathematikunterricht in einem begrenzten Setting zu untersuchen.

Methodisch hat sich außerdem der Vorteil der Verwendung der Schätzung der Aufgabenschwierigkeiten durch das LLTM gezeigt. Wie bereits in Kapitel 8 erwähnt, hätte die Schätzung auch ausschließlich durch Regressionsmodelle erfolgen können. Die Verwendung des LLTM zur Schätzung der Aufgabenschwierigkeiten für den Zweck dieser Arbeit hatte aber den Vorteil, dass die Aufgabenschwierigkeiten nicht nur durch das Rasch-Modell evaluiert und als abhängige Variable in das Regressionsmodell eingebracht wurden, sondern dass genau spezifiziert werden konnte, welche Schätzung für welche einzelnen Aufgaben geeignet war. Dadurch konnte demonstriert werden, für welche Aufgaben eine Schätzung durch die Faktoren gelungen ist und für welche weniger. Durch diese Erkenntnisse konnte demonstriert werden, dass das Instrument die Aufgabenschwierigkeiten für Aufgaben mit höheren Anforderungen deutlich genauer vorhersagt und dass genau bestimmt wird, welche Aufgaben das sind. Denkbar wäre diesbezüglich eine stoffdidaktische und weitergehende sprachliche Analyse, die jedoch nicht Ziel der Arbeit war. So konnten die Schätzung der Aufgabenschwierigkeit und die Bestimmung des Effektes auf die Aufgabenschwierigkeit zeigen, dass die Kombination aus IRT-Methoden und weiteren statistischen Verfahren zwar umfangreich ist, jedoch eine hohe Erklärungsleistung aufweist.

Diese Arbeit verdeutlicht außerdem, dass die Verbindung von strukturentdeckenden (multivariaten) Verfahren, beispielsweise der explorativen Faktorenanalyse, der Clusteranalyse oder der Korrespondenzanalyse, und eine anschließende qualitative Folgeuntersuchung eine produktive Möglichkeit einer Nutzung eines Mixed-Methods-Ansatzes bietet (Kleine \& Jordan, 2013). Durch die Verknüpfung von quantitativer und qualitativer Untersuchung können der Mehrwert der quantitativen Analyse und die Güte der Typenbildung für die qualitative Vertiefungsanalyse deutlich erhöht und je nach Forschungsanliegen können unterschiedliche Forschungsaspekte in der qualitativen Untersuchung forciert werden, beispielsweise auch eine stoffdidaktische Analyse.

Speziell für die sprachliche Untersuchung von mathematischer Kommunikation im Unterricht bietet sich das in dieser Arbeit gezeigte Verfahren an, Register auf jeder Ebene der Generalität zu analysieren. Die Verbindung von quantitativem und qualitativem Verfahren eignet sich wie gezeigt dazu, die Verbindung zwischen sprachlichen Faktoren und damit assoziierten fachlich-kontextuellen Merkmalen zu untersuchen. 
Open Access Dieses Kapitel wird unter der Creative Commons Namensnennung 4.0 International Lizenz (http://creativecommons.org/licenses/by/4.0/deed.de) veröffentlicht, welche die Nutzung, Vervielfältigung, Bearbeitung, Verbreitung und Wiedergabe in jeglichem Medium und Format erlaubt, sofern Sie den/die ursprünglichen Autor(en) und die Quelle ordnungsgemäß nennen, einen Link zur Creative Commons Lizenz beifügen und angeben, ob Änderungen vorgenommen wurden.

Die in diesem Kapitel enthaltenen Bilder und sonstiges Drittmaterial unterliegen ebenfalls der genannten Creative Commons Lizenz, sofern sich aus der Abbildungslegende nichts anderes ergibt. Sofern das betreffende Material nicht unter der genannten Creative Commons Lizenz steht und die betreffende Handlung nicht nach gesetzlichen Vorschriften erlaubt ist, ist für die oben aufgeführten Weiterverwendungen des Materials die Einwilligung des jeweiligen Rechteinhabers einzuholen. 